Revista de Investigación de Física 23(3), (2020)

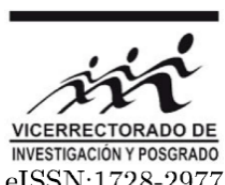

\title{
Ubicación de la mayor concentración de nieblas de advección en la Costa Central del Perú entre los años 2000 -2014, usando imágenes satelitales, como potenciales recursos de agua dulce
}

\author{
Tatiana León ${ }^{12^{*}}$, Leónidas Ocola ${ }^{1}$, Joel Rojas ${ }^{1}$ \\ ${ }^{1}$ Universidad Nacional Mayor de San Marcos, Facultad de Ciencias Fisicas \\ ${ }^{2}$ Universidad Nacional de Ingeniería, Facultad de Ciencias
}

Recibido 22 abril 2020 - Aceptado 12 diciembre 2020

\begin{abstract}
Resumen
Las nieblas forman la vegetación de las lomas, las cuales son cosechadoras naturales de agua de niebla, las zonas de mayor vegetación, son las zonas de mayor concentración de niebla. Una característica importante en las lomas es que la altitud es el predictor más importante de su riqueza vegetal, es por esto que para encontrar las mayores concentraciones de niebla se analiza la vegetación y la altitud. Los datos satelitales que dan información de vigor vegetal y altitud de las lomas son: el Índice Normalizado de Vegetación (NDVI) y el Modelo de Elevación Digital (DEM), con estos datos se localiza la vegetación de las lomas, luego se analiza la correlación entre NDVI y altitud a través de perfiles topográficos que atraviesan las Lomas más importantes de la costa. En los resultados se observa que el NDVI aumenta al aumentar la altitud llegando a un punto máximo a partir del cual el NDVI disminuye al seguir aumentando la altitud, a mayor valor de NDVI mayor disponibilidad de agua de las nieblas. Se analizaron seis lomas distribuidas a lo largo de la Costa Central del Perú, dando como resultado que el máximo desarrollo de la vegetación dentro de una loma está a una altura promedio de $644.6 \pm 101.8 \mathrm{msnm}$, altura donde está la mayor concentración de niebla, lugar donde se puede instalar atrapanieblas para colectar agua.
\end{abstract}

Palabras Clave: Niebla de advección, lomas, NDVI, altitud, perfil topográfico.

\section{Location of the highest concentration of advection fogs on the Central Coast of Peru between the years 2000 -2014, using satellite images, as potential freshwater resources}

\begin{abstract}
The fogs form the vegetation of the Lomas, which are natural harvesters of fog water, the areas with the greatest vegetation, are the areas with the highest concentration of fog. An important characteristic in the Lomas is that altitude is the most important predictor of its plant richness, therefore to find the places with highest concentrations of fog, vegetation, and altitude are analyzed. The satellite data that give information on plant vigor and altitude of the Lomas are: the Normalized Vegetation Index (NDVI) and the Digital Elevation Model (DEM), with these data the vegetation of the Lomas, is located, then the correlation is analyzed between NDVI and altitude through topographic profiles that cross the most important Lomas of the coast. The results show that the NDVI increases with increasing altitude, reaching a maximum point from which the NDVI decreases as the altitude continues to increase, the higher the NDVI value, the greater the availability of water from the fog. Six Lomas distributed along the Central Coast of Peru were analyzed, resulting in that the maximum development of vegetation within a Loma is at an average height of $644.6 \pm 101.8$ masl, where it is the highest concentration of fog, a place where you can install fog catchers to collect water.
\end{abstract}

KeyWords: Advection fog, lomas, NDVI, altitude, topographic profile.

1 tleonr@uni.pe 


\section{Introducción}

Las nieblas de advección océano-continente son recursos hídricos alternativos de agua dulce aún sin explotar en la región geográfica de la costa peruana. Su potencial, como recurso de agua dulce, se ha demostrado, en varios países, mediante la acumulación de agua por atrapanieblas para usos múltiples. Las nieblas abastecen de agua, entre otros ambientes, a las lomas costeras (Del Río et al., 2018). Una característica importante de la vegetación en las lomas es que la altitud es el predictor más importante de su riqueza vegetal, por el aumento de la condensación de agua con la altitud, hasta llegar a la zona de inversión térmica. La mayor humedad se encuentra por debajo de esta zona de inversión. Para evaluar esta riqueza vegetal se utiliza el índice de vegetación (NDVI, sigla en inglés).

El NDVI es el índice de vegetación que provee una medida del vigor, salud, y distribución de la vegetación en una superficie dada y un tiempo de observación. Es simple de calcular, eficiente, fácil de obtener de registros de sensores remotos, y muy utilizado. La salud de la vegetación depende de la naturaleza y humedad del suelo, entre otros factores. La niebla y vapor de agua atmosférico del océano al ascender por las colinas, por acción del viento, y disminuir la temperatura atmosférica con la altitud, el vapor de agua se condensa al alcanzar el punto de rocío, las gotas microscópicas de agua de la niebla coalescen, y se depositan, en ambos casos como agua líquida, en la superficie del terreno y/o hojas de la vegetación.

Esta agua termina en el suelo y contribuye al desarrollo, vigor y salud de la vegetación, como demuestra la correlación positiva entre los valores del NDVI. y la correspondiente elevación del DEM (modelo digital de elevación). Las zonas con mayor vegetación corresponden a lugares con mayor disponibilidad de agua del suelo proveniente de la niebla, ya que, no hay agua de lluvia. Las lomas costeras son las "cosechadoras" naturales del agua de niebla en la costa peruana.

Con las imágenes satelitales, se delimita la costa central del Perú y se discrimina la vegetación producida por ríos y áreas de cultivo para obtener un mapa con sólo vegetación natural de lomas, luego se crean perfiles topográficos que atraviesan las lomas más importantes de la costa finalmente se halla el grado de correlación entre el NDVI y la altitud. El análisis de esta correlación arroja diversos resultados entre los más importantes está la obtención del umbral NDVI de formación de lomas en la costa central del Perú igual a 0.26 , ubicado a una altura promedio de $259 \pm 97.27$ msnm, otro resultado importante es la altura máxima promedio de las lomas igual a $1033 \pm 100.06 \mathrm{msnm}$ y entre estos dos limites se encuentra que el mayor desarrollo de la vegetación se da a una altura promedio de $644.6 \pm$ $101.8 \mathrm{msnm}$, altura donde está la mayor concentración de nieblas de advectión, lugar donde se puede instalar atrapanieblas para colectar agua.

\section{Metodología}

Se adquieren dos tipos de datos satelitales, el índice normalizado de vegetación (NDVI) y el modelo de elevación digital (DEM). El NDVI tiene como nombre corto MOD13Q1 este dato es adquirido por el sensor MODIS que está abordo del satélite Terra, tiene un nivel de procesamiento 3, resolución temporal de 16 días, resolución espacial de $250 \mathrm{~m}$. El MOD13Q1 da información del mismo lugar los días julianos 1, 17, 33, 49, 65, 81, 97, 113, 129, 145, 161, 177, 193, 209, 225, 241, $257,273,289,305,321,337$ y 353, siendo un total de 23 imágenes por año. Para obtener información de NDVI del Perú se tiene que elaborar un mosaico con 5 imágenes satelitales por cada día juliano, requiriéndose en total 115 imágenes por año. En esta investigación se toman datos por 15 años desde el 2000 hasta el 2014, empleando un total de 1725 datos satelitales que se transforman de 342 imágenes NDVI de Perú, estos datos fueron descargados de la página web "MODIS Reprojection Tool Web (MRTweb), mientras que el modelo de elevación digital DEM que da información topográfica, es obtenido de Shuttle Radar Topographic Mission (SRTM), descarga de la página web "Consortium for Spatial Information" Figura 1.

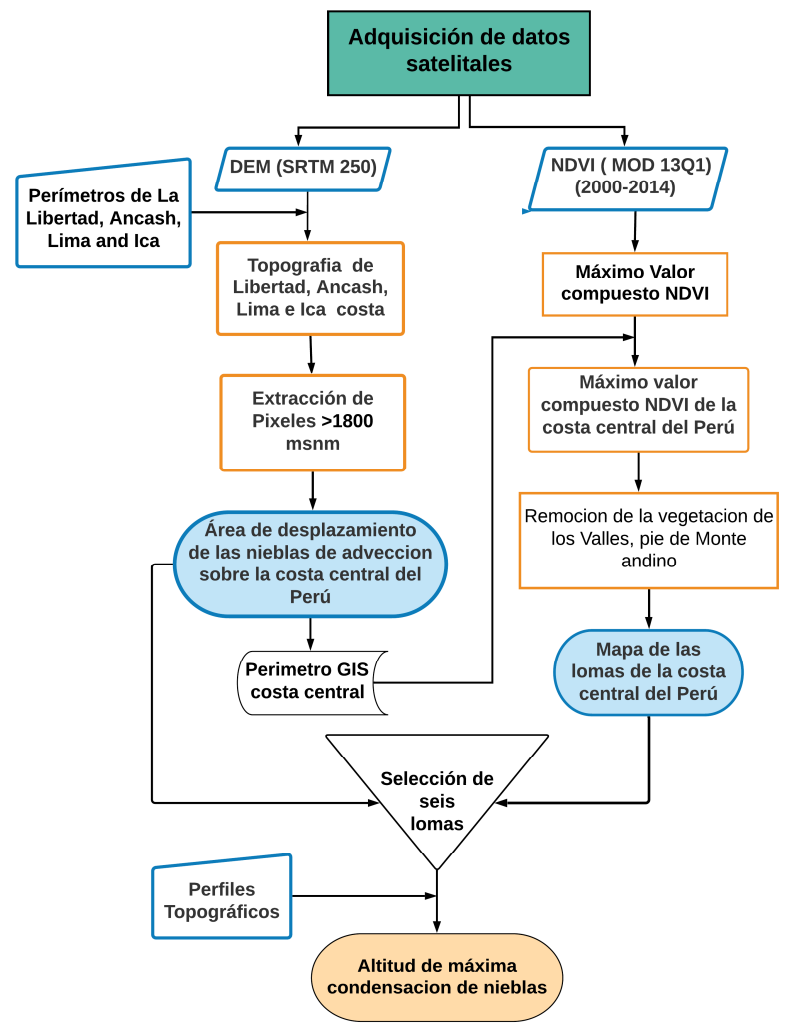

Figura 1.Metodología de Investigación 


\section{Resultados}

\section{Obtención del área de desplazamiento de las nieblas de advección en la Costa Central del Perú}

La capa de inversión térmica en la costa peruana está aprox. entre 750 y $1800 \mathrm{msnm}$ (León,2017), esta capa restringe el ascenso de las nieblas, una vez que estas ingresan a la costa se difunden tierra adentro sobre un terreno relativamente plano, hasta llegar a zonas donde las montañas se elevan abruptamente, a medida que van ascendiendo la condensación aumenta con la altitud hasta alcanzar el límite inferior de la capa de inversión térmica (Muenchow et al., 2013), alrededor de estas regiones las nieblas se concentran y su humedad permite la formación de comunidades biológicas llamadas lomas (Veblen et al., 2007).

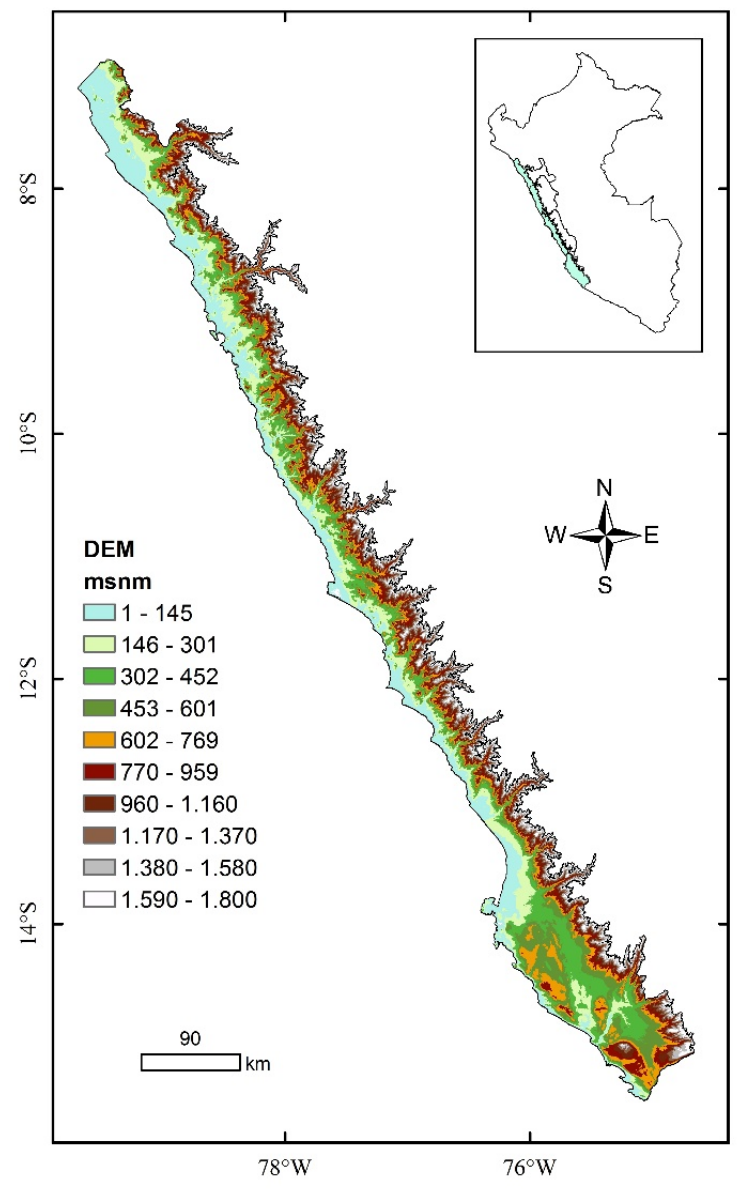

Figura 2.Topografía de la costa central del Perú, área que va de 0 - $1800 \mathrm{msnm}$, en esta región se desplazan las nieblas de advección, los colores indican niveles de altitud.

Se construye un área considerando que la capa de inversión esté dentro ya que es la limita el desplazamiento vertical de las nieblas, para esto se toman la imagen satelital
SRTM250 y los perímetros oficiales de los departamentos de la libertad, Ancash, Lima e Ica, esta imagen es recortada con un perímetro que es la fusión de los limites departamentales, a la imagen resultante se le retira todos los pixeles mayores a $1800 \mathrm{msnm}$, con esto se obtiene la topografía de la costa central del Perú lugar donde se desplazan las nieblas de advección, Figura 2.El contorno del mapa topográfico será utilizado delimitar las imágenes satelitales de NDVI del Perú.

\section{Identificación de las áreas de condensación de niebla en la Costa Central Perú}

El efecto que produce la niebla al ingresar a la costa son las lomas, para encontrar las regiones donde hay mayor condensación, se tiene que encontrar la vegetación de las lomas, para esto el primer paso es elaborar un mapa máximo valor compuesto NDVI de la costa central del Perú, usando datos de NDVI (MOD13Q1) por un periodo de 15 años (2000-2014), esto da como resultado, un mapa que muestra el área máxima de vegetación desarrollada por los ríos (Valles) y por las nieblas (lomas) entre el 2000 al 2014 Figura 3a. Se retira toda la vegetación de los valles (León, 2017) y quedan pequeños grupos de vegetación, de los cuales sólo se selecciona la vegetación que está en medio del desierto y a través del software ArcGis se crea perímetros para delimitar a esta vegetación Figura3b. Utilizando Google Earth pro y diversas fuentes bibliográficas como: (Veblen et al., 2007), (Beresford et al., 2015), (Trujillo et al., 2011) y (Rundel et al., 1991), se comprueba que dentro de estos perímetros están las lomas costeras, en base a este resultado se concluye que las regiones delimitadas son las áreas de condensación de nieblas.

Altitud a la que se produce la máxima condensación dentro de una loma costera de la costa central del Perú

En una loma la condensación incrementa al incrementar la altitud, esto significa que a diferentes niveles altitudinales diferente disponibilidad de agua, y la mayor disponibilidad de agua se encuentra bajo la capa de inversión térmica, en estas condiciones la vegetación de las lomas se desarrolla en diferentes cinturones, cuya composición está basada en la disponibilidad de humedad, que es predominante en función de la altitud. A parte de la altitud hay otros factores que influyen en la riqueza de la vegetación como son la pendiente, el aspecto, el tipo de superficie del suelo, la intensidad, dirección y velocidad del viento; pero la altitud es el predictor más importante en la riqueza y distribución de las especies en la loma. (Muenchow, et al., 2013), es por esto que se analiza la relación entre los parámetros NDVI y Altitud para encontrar los lugares de máxima condensación de niebla. Para sacar un valor promedio de la altura en la que se da la máxima condensación, se analiza en seis lomas la relación entre NDVI y altitud, para esto se crean perfiles topográficos 
Rev. Inv. Fís. 23(3), (2020)

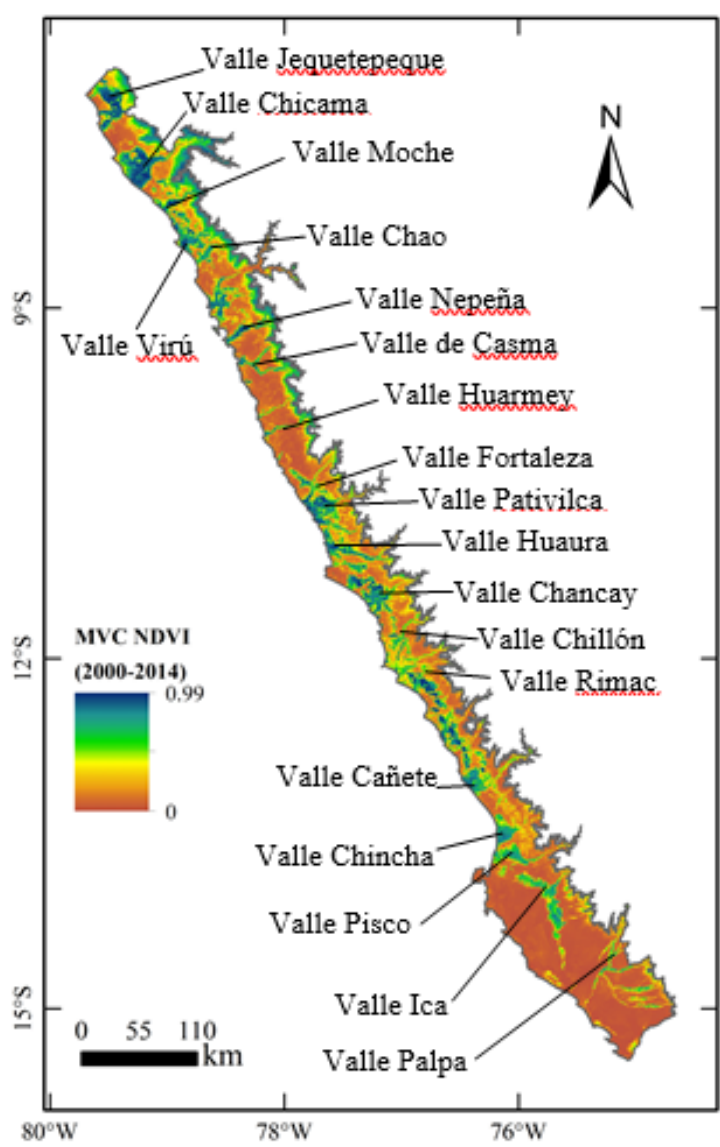

(a)

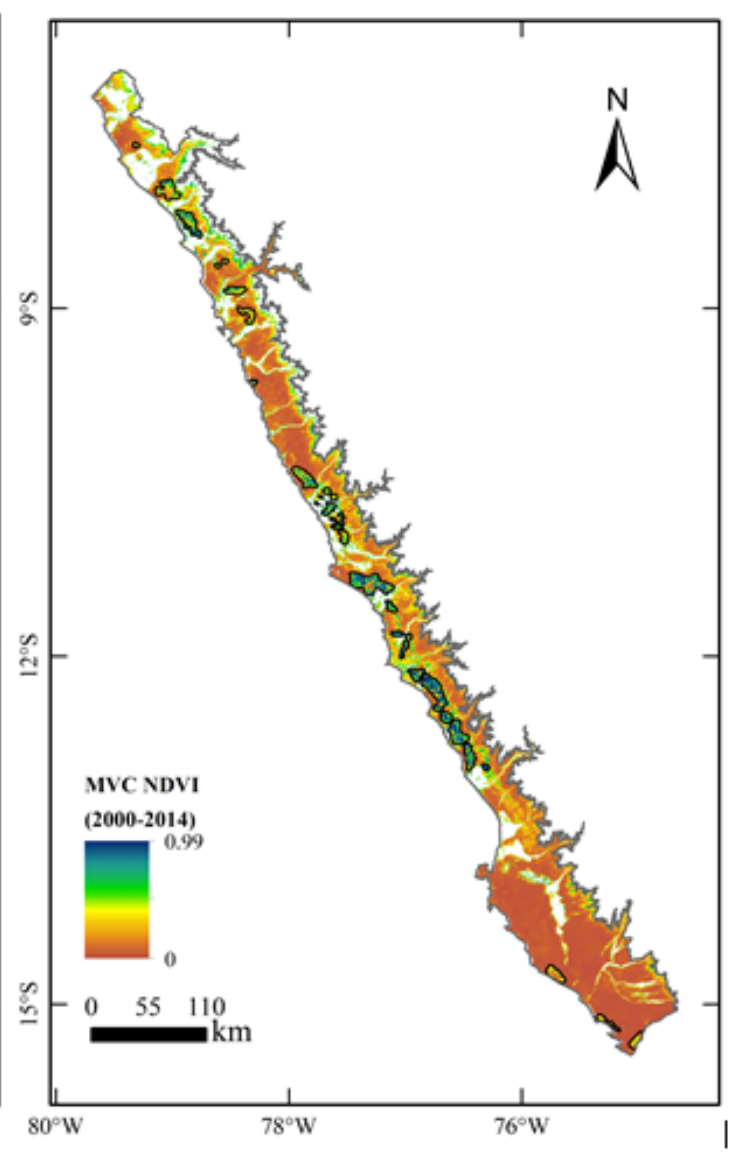

(b)

Figura 3. Mapas máximo valor compuesto NDVI (2000-2014) de la costa central del Perú, los valores cerca de 0.9 indican abundante vegetación y los valores cerca a cero indican desierto (a) muestra toda la vegetación desarrollada entre los años 2000-2014, no se puede diferenciar entre la vegetación de los valles y las lomas (b) muestra la costa central sin valles, las regiones delimitadas son áreas de condensación de niebla que dan lugar a la formación de lomas.

que inician en la línea de costa y finalizan al atravesar la loma Figura 4

- $\quad$ En la costa de La Libertad: Loma cerro Campana: Perfil AB (A: $\left.8.0^{\circ} \mathrm{S}, 79.1^{\circ} \mathrm{W}, \mathrm{B}: 7.9^{\circ} \mathrm{S}, 79.1^{\circ} \mathrm{W}\right)$, Loma cerro Chiputur: Perfil CD $\left(\mathrm{C}: 8.3^{\circ} \mathrm{S}\right.$, $\left.78.9^{\circ} \mathrm{W}, \mathrm{D}: 8.1^{\circ} \mathrm{S}, 78.9^{\circ} \mathrm{W}\right)$;

- En la costa de Ancash: Loma cerro Mongón: Perfil EF: (E: $\left.9.7^{\circ} \mathrm{S}, 78.3^{\circ} \mathrm{W}, \mathrm{F}: 9.6^{\circ} \mathrm{S}, 78.3^{\circ} \mathrm{W}\right)$,
- $\quad$ Loma cerro Lupín: Perfil IK: (I: $10.5^{\circ} \mathrm{S}, 77.9 \mathrm{~W}$ y en $\left.\mathrm{K}: 10.4^{\circ} \mathrm{S}, 77.8^{\circ} \mathrm{W}\right)$;

- En la costa de Lima: Loma de Lachay: Perfil RS (R $11.5^{\circ} \mathrm{S}, 77.4^{\circ} \mathrm{W}$ y en $\left.\mathrm{S}: 11.3^{\circ} \mathrm{S}, 77.4^{\circ} \mathrm{W}\right)$;

- En la costa de Ica: Loma de Amará: Perfil PZ (P: $14.7^{\circ} \mathrm{S}, 75.8^{\circ} \mathrm{W}$ y $\left.\mathrm{Z}: 14.7^{\circ} \mathrm{S}, 75.7^{\circ} \mathrm{W}\right)$. 
Rev. Inv. Fís. 23(3), (2020)

(a)

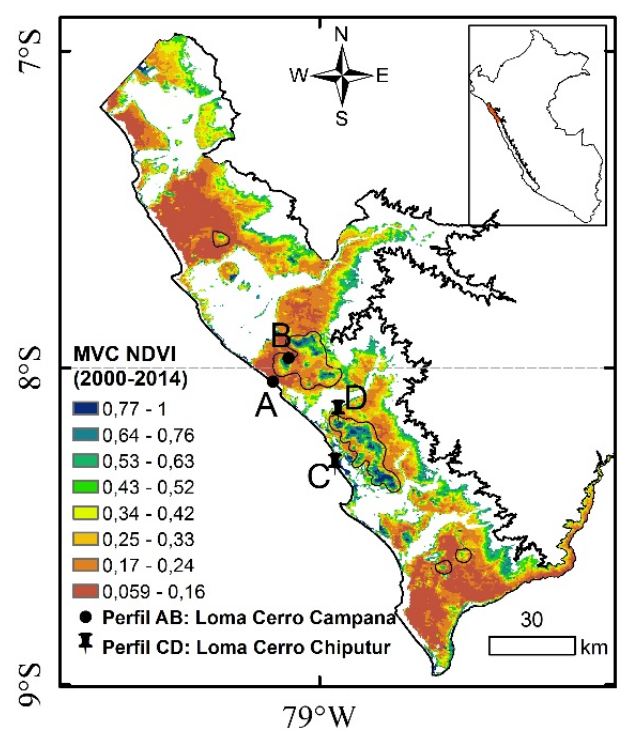

(c)

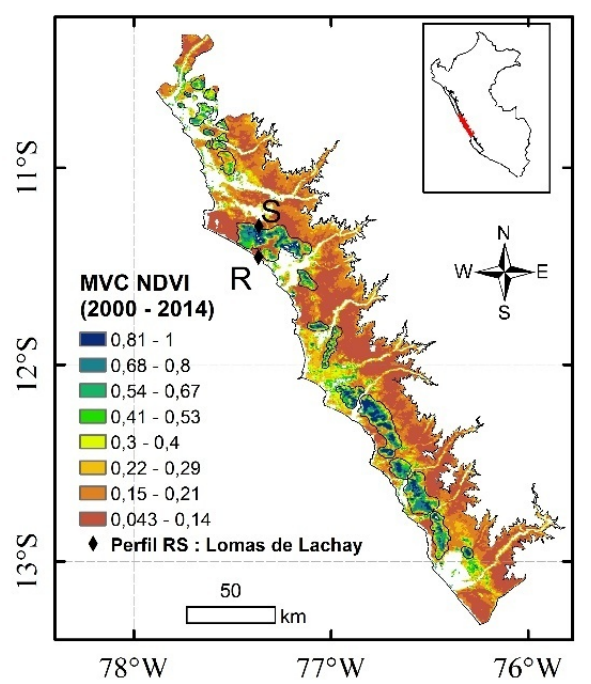

(b)

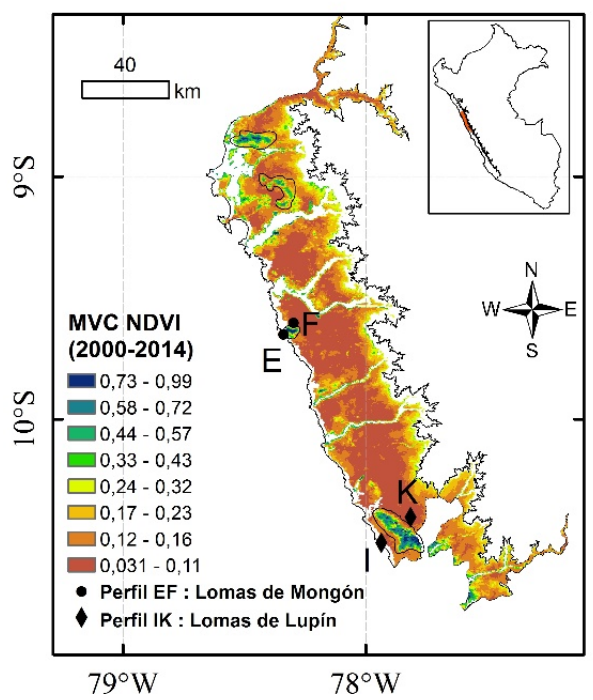

(d)

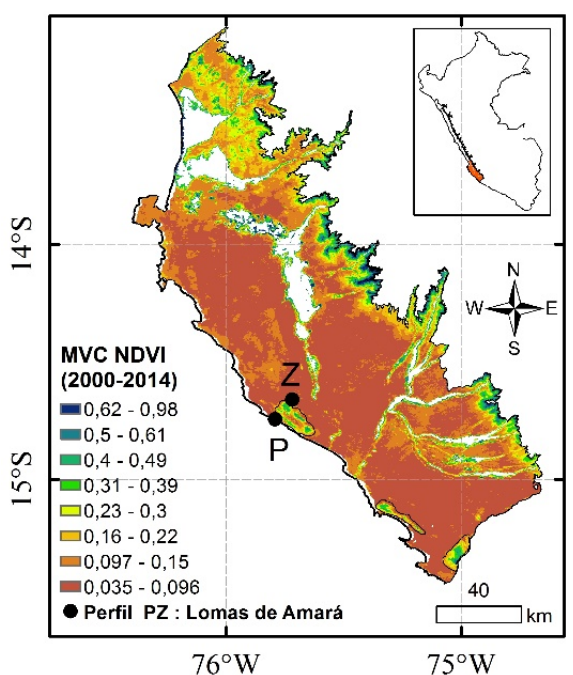

Figura 4. Mapas Máximo valor compuesto NDVI (2000-2014) de la Costa Central de Perú, sin vegetación rivereña dividido por departamentos: (a) Costa de La Libertad, (b) Costa de Ancash, (d) Costa de Lima y (d) Costa de Ica, con sus respectivos perímetros de condensación y perfiles topográficos que atraviesan una loma costera (AB: Loma Cerro Campana, CD: Loma Chiputur, EF: Loma Mongón, IK: Loma Lupín, RS: Loma Lachay y PZ: loma Amará).

\section{Perfil topográfico AB en la loma de Cerro Campana}

Un perfil topográfico es un conjunto de coordenadas trazadas en lo posible de forma perpendicular a la topografía de una loma, por cada punto del perfil se recoge información de NDVI y altitud, por ejemplo: la loma Cerro Campana se encuentra dentro del perímetro de condensación de la costa de La Libertad. En los mapas MVC-NDVI MOD13Q1 (2000-2014) y Mapa topográfico
(SRTM 250) que contienen a esta loma, se traza el perfil $\mathrm{AB}$, este perfil inicia en la línea de costa $\mathrm{A}\left(8.0^{\circ} \mathrm{S}, 79.1^{\circ} \mathrm{W}\right)$ y finaliza pasando toda la vegetación de la loma en el punto B $\left(7.9^{\circ} \mathrm{S}, 79.1^{\circ} \mathrm{W}\right)$, como se muestra Figuras 5a y $5 b$. A través de cada punto del perfil topográfico se recoge información de NDVI y altitud lo que se grafica en una sola ventana como se muestra en la Figura.6a luego se halla la relación NDVI vs Altitud Figura 6b. 
Rev. Inv. Fís. 23(3), (2020)

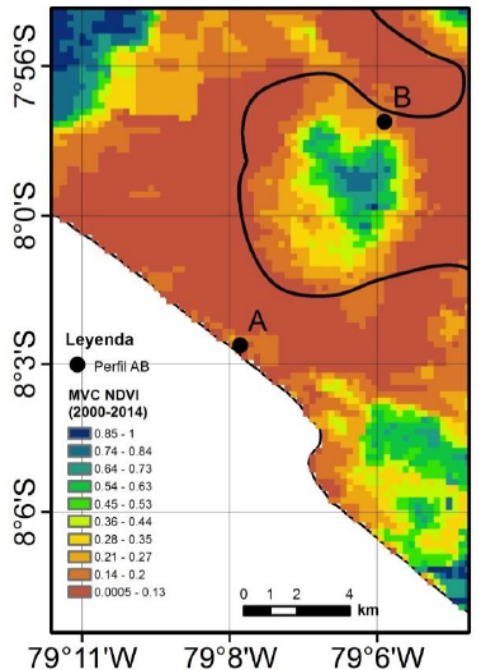

(a)

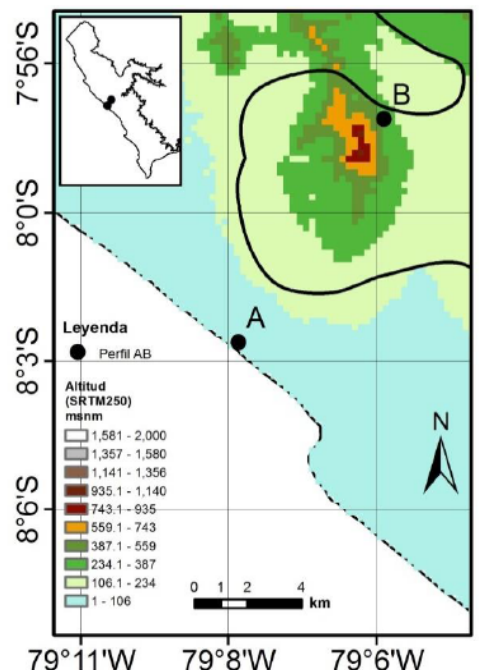

(b)

Figura 5.Mapas Máximo valor compuesto NDVI (2000-2014) y topográfico (SRTM250) de la Loma Cerro Campana con sus respectivos perfiles $\mathrm{AB}, \mathrm{A}:\left(8.0^{\circ} \mathrm{S}, 79.1^{\circ}\right) \mathrm{W}$ y en $\mathrm{B}:\left(7.9^{\circ} \mathrm{S}, 79.1^{\circ} \mathrm{W}\right)$, donde los colores indican valores de NDVI y niveles de altitud.

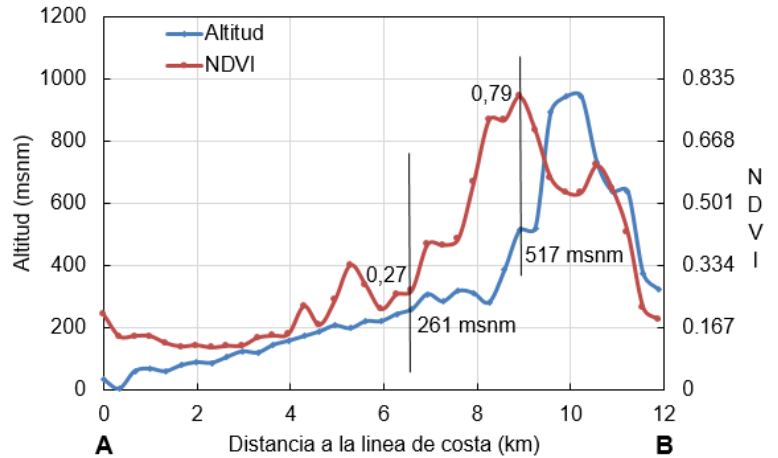

(a)

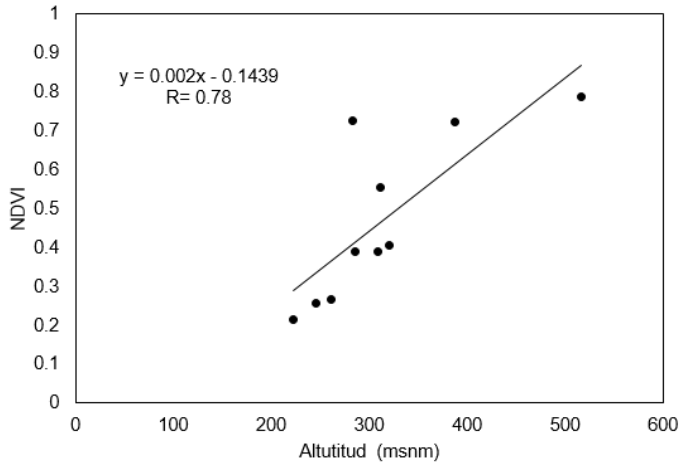

(b)

Figure 6.(a) Variación del NDVI con la altitud, a través del perfil AB que pasa por la loma de Cerro Campana, en azul: la variación de la altitud entre los puntos $\mathrm{AB}$ de la Figura 5a, en rojo: la variación del NDVI entre los puntos $\mathrm{AB}$ de la Figura 5b. Las líneas verticales indican la región en la que el NDVI incrementa a medida que aumenta la altitud. (b) Relación del NDVI vs. Altitud, obtenida del perfil AB que pasa por la loma Cerro Campana, donde el grado de relación entre la altitud y el NDVI es 0.78 .

En esta grafica se observa, que a una distancia aproximada de $6.5 \mathrm{~km}$ de la línea de costa (punto A), a una altura de 261 msnm y con un valor de NDVI igual a 0.27 , los valores de NDVI comienzan a aumentar bruscamente a medida que aumenta la altitud, hasta alcanzar un valor máximo de 0.79 que corresponde a una altitud aproximada de 517 msnm, a partir de este punto, el NDVI empieza a disminuir aun cuando sigue aumentando la altitud. En el punto donde comienza a incrementar bruscamente el NDVI con la altitud, el valor que toma el NDVI, es el valor del umbral NDVI de formación de la loma Cerro Campana, el cual equivale a $0.27 \mathrm{y}$ el punto donde el NDVI toma su máximo valor, corresponde al lugar donde se da mayor condensación de niebla.El grado de relación entreel NDVI y la altitud en la loma de Cerro Campana es de 0.78 , que es calculado desde que el NDVI toma el valor del umbral hasta el punto en que el NDVI cae drásticamente Figura. 7b. lo que indica que el NDVI varia directamente proporcional con la altitud.El procedimiento aplicado a la loma Cerro Campana es aplicado a las demás lomas escogidas, lo que se puede resumir en la siguiente tabla1. 
Rev. Inv. Fís. 23(3), (2020)

Tabla 1. Principales lomas de la Costa Central del Perú donde se presenta seis características físicas obtenidas de la relación NDVI vs altitud

\begin{tabular}{|c|c|c|c|c|c|c|c|}
\hline $\begin{array}{l}\text { Departa } \\
\text { mentos }\end{array}$ & Lomas & $\begin{array}{c}\text { Umbral } \\
\text { NDVI }\end{array}$ & $\begin{array}{c}\text { Altura del } \\
\text { umbral } \\
(\mathrm{msnm})\end{array}$ & $\begin{array}{c}\text { Máximo } \\
\text { valor de NDVI } \\
\text { encontrado }\end{array}$ & $\begin{array}{c}\text { Altura del } \\
\text { máximo valor } \\
\text { de NDVI } \\
\text { (msnm) }\end{array}$ & \begin{tabular}{|c|} 
Grado de \\
correlación \\
NDVI y \\
DEM \\
\end{tabular} & $\begin{array}{c}\text { Max altura } \\
\text { de la loma }\end{array}$ \\
\hline \multirow{2}{*}{ La Libertad } & $\begin{array}{l}\text { Campana } \\
\text { Perfil AB }\end{array}$ & 0.27 & 261 & 0.79 & 517 & 0.8 & 945 \\
\hline & $\begin{array}{l}\text { Chiputur } \\
\text { Perfil CD }\end{array}$ & 0.24 & 349 & 0.92 & 674 & 0.9 & 1055 \\
\hline \multirow{2}{*}{ Ancash } & $\begin{array}{l}\text { Mongón } \\
\text { Perfil EF }\end{array}$ & 0.22 & 204 & 0.86 & 572 & 0.9 & 961 \\
\hline & $\begin{array}{c}\text { Lupin } \\
\text { Perfil IK }\end{array}$ & 0.26 & 283 & 0.78 & 777 & 0.8 & 1195 \\
\hline Lima & $\begin{array}{c}\text { Lachay } \\
\text { Perfil RS }\end{array}$ & 0.31 & 359 & 0.89 & 683 & 0.7 & 1013 \\
\hline Promedio & & $0.26 \pm 0.03$ & $259 \pm 58.97$ & $0.85 \pm 0.06$ & $644.6 \pm 101.8$ & $0.8 \pm 0.083$ & $1033.8 \pm 100.06$ \\
\hline Ica & $\begin{array}{c}\text { Amará } \\
\text { Perfil PZ }\end{array}$ & $\begin{array}{c}\text { Sin } \\
\text { definir }\end{array}$ & - & 0.39 & 719 & 0.8 & \\
\hline
\end{tabular}

\section{Conclusiones}

El principal resultado de esta investigación fue el desarrollo de un mapa, que muestra los lugares donde se produjo las mayores condensaciones de niebla a lo largo de la costa central del Perú.Esta metodología se puede aplicar a lo largo de toda la costa peruana y aplicar a diferentes periodos de tiempo lo que permite implementar nuevos proyectos a escala regional, para aprovechar los suministros adicionales de recursos hídricos.En las costas de los departamentos de la Libertad, Ancash y Lima la altura promedio recomendable para colocar atrapanieblas y poder cosechar el agua es $644.6 \pm 101.8 \mathrm{msnm}$, mientras que en la costa de Ica no se recomienda implementar proyectos de atrapanieblas.

\section{Agradecimientos}

Agradecemos a la facultad de Ciencias Físicas - UNMSM, en particular al Al Grupo de Investigación Física de los Cambios Ambientales y Teledetección (LABTEL) por las facilidades y el apoyo brindado en el desarrollo del presente trabajo de investigación.

\section{Referencias}

Beresford-Jones, D., Pullen, A. G., Whaley, O. Q., Moat,J., Chauca, G., Cadwallader, L.,French, C. (2015). Reevaluating the resource potential of lomas fog oasis environments for Preceramic hunter-gatherers under past ENSO modes on the south coast of Peru. Quaternary Science Reviews, 129,196215.

https://doi.org/10.1016/j.quascirev.2015.10.025.

Del Río, C., Garcia, J. L., Osses, P., Zanetta, N., Lambert, F., Rivera, D., ... Lobos, F. (2018). ENSO influence on coastal fog-water yield in the atacama desert, Chile. Aerosol and Air Quality Research, 18(1), 127-144. https://doi.org/10.4209/aaqr.2017.01.0022

Muenchow, J., Bräuning, A., Rodríguez, E. F., \& von Wehrden, H. (2013). Predictive mapping of species richness and plant species' distributions of a peruvian fog oasis along an altitudinal gradient. Biotropica, 45(5), 557-566. https://doi.org/10.1111/btp.12049
Muenchow, J., Hauenstein, S., Bräuning, A., Bäumler, R., Rodríguez, E. F., \& Von Wehrden, H. (2013). Soil texture and altitude, respectively, largely determine the floristic gradient of the most diverse fog oasis in the Peruvian desert. Journal of Tropical Ecology, 29(5), 427-438. https://doi.org/10.1017/S0266467413000436

Rundel, P., Dillon, M., \& Palma, B. (1991). The phytogeography and ecology of the coastal Atacama and peruvian deserts.ALISO, 1-49.

Trujillo, D., \& Delgado Rodríguez, A. (2011). Aa from lomas formations. A new Orchidaceae record from the desert coast of Peru. Lankesteriana, 11(1), 33-38. https://doi.org/10.15517/lank.v11i1.18313

Veblen, T., Young, K., \& Orme, A. (2007). The Physical Geography of South America.Oxford: Oxford University Press.

León, T. (2017). La climatología de las lomas de la Costa Central del Perú usando datos imágenes de satélite. Tesis de maestría Lima: Universidad Nacional Mayor de San Marcos. 